\title{
CFD Analysis of an Installation Used to Measure the Skin-Friction Penalty of Acoustic Treatments
}

\author{
Philippe R. Spalart ${ }^{1}$ \\ Boeing Commercial Airplanes, Seattle, WA, 98124 \\ Andrey Garbaruk ${ }^{2}$ \\ St.-Petersburg Polytechnic University, St.-Petersburg, 195220, Russian Federation \\ Brian M. Howerton ${ }^{3}$ \\ NASA Langley Research Center, Hampton, VA, 23681
}

\begin{abstract}
There is a drive to devise acoustic treatments with reduced skin-friction and therefore fuel-burn penalty for engine nacelles on commercial airplanes [1]. The studies have been experimental, and the effects on skin-friction are deduced from measurements of the pressure drop along a duct. We conduct a detailed CFD analysis of the installation, for two purposes. The first is to predict the effects of the finite size of the rig, including its nearsquare cross-section and the moderate length of the treated patch; this introduces transient and blockage effects, which have not been included so far in the analysis. In addition, the flow is compressible, so that even with homogeneous surface conditions, it is not homogeneous in the streamwise direction. The second purpose is to extract an effective sandgrain roughness size for a particular liner, which in turn can be used in a CFD analysis of the aircraft, leading to actual predictions of the effect of acoustic treatments on fuel burn in service. The study is entirely based on classical turbulence models, with an appropriate modification for effective roughness effects, rather than directly modeling the liners.
\end{abstract}

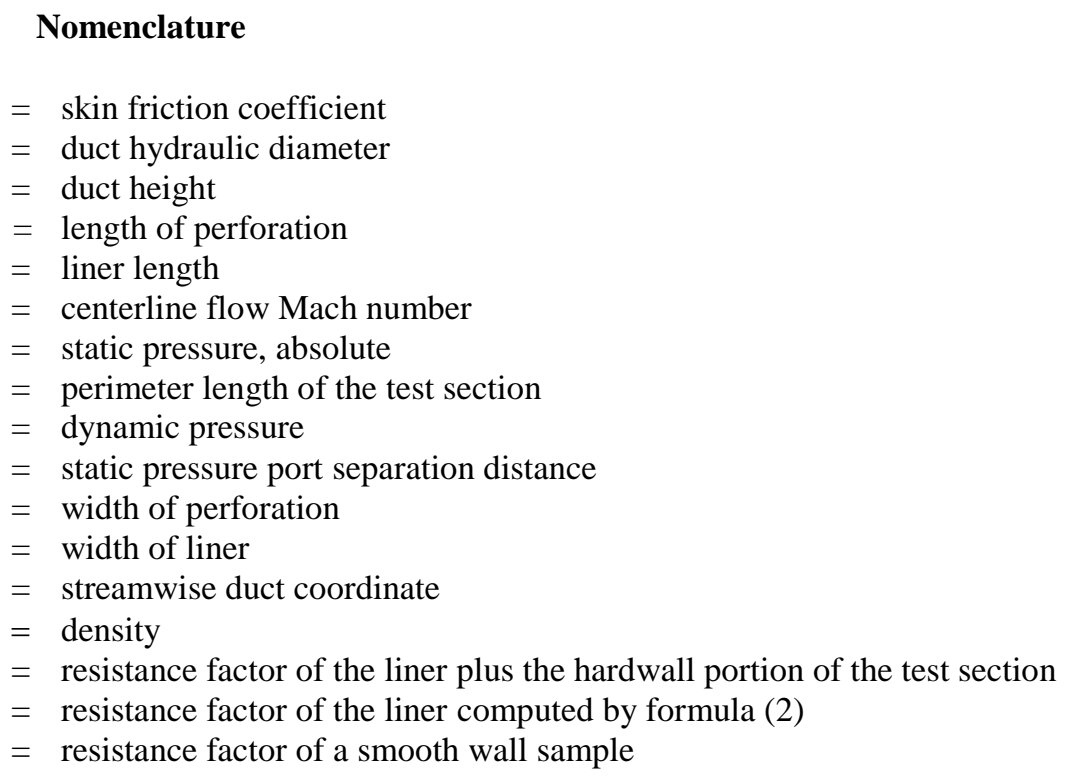

\footnotetext{
${ }^{1}$ Senior Technical Fellow, Senior Member AIAA.

${ }^{2}$ Senior Research Scientist.

${ }^{3}$ Research Scientist, Structural Acoustics Branch, MS 463, Senior Member AIAA.
} 


\section{Introduction}

$\mathrm{T}$

The NASA Langley Grazing Flow Impedance Tube (GFIT) is used for creative design and testing of acoustic liners in the presence of grazing flow [1,2]. Recent efforts have added drag measurement capabilities, which have been employed to develop liners that appear to suppress their typical skin-friction penalty. This could have a significant value to the airline industry in terms of reduced fuel burn, especially considering the large wetted area of modern fan ducts. Other incentives to create and test new liners include manufacturing cost, and acoustic performance. The facility also allows the imposition of strong acoustic tones, which increase the momentum loss at the surface, and emulate the situation in an engine inlet with the rotating pressure field of the fan. This is, however, not addressed by the present, steady calculations which treat the liner behavior as an effective roughness via modifications of the turbulence model [3,4]. Predictive simulations of a turbulent boundary layer over the liner geometry including such tones, by Direct Numerical Simulation or possibly Large-Eddy Simulation techniques, are a distant prospect because of the extreme complexity of the geometry that would be needed, with a very large number of facesheet perforations. Depending on the relative sizes of the perforations and boundary-layer thickness, two-step modeling with a porous-surface boundary condition (averaging over a large number of perforations but including the cavities) might be an option. This would be a separate, and far more ambitious modeling and simulation effort.

The objective of the current study is to investigate use of NASA's static pressure drop method to deduce liner drag. Due to the finite size of the duct and the relatively modest length of the liner treatment, there is concern about the effect of pressure transients and blockage on the results. The compressible nature of the flow in the GFIT (with streamwise Mach numbers above 0.3) adds variability that must be accounted for. Ideally, one could model the liner as additional, distributed sand-grain roughness educed from matching experimental pressure data. Such a result could be used in calculations to predict the effect of the installed acoustic treatment on overall aircraft drag.

\section{Problem Definition and CFD Approach}

Figure 1 presents the geometry of the computational domain, reproducing the GFIT geometry. The entry region is long enough for the boundary layers to grow and completely invade the potential core, but without quite establishing a fully-developed turbulent duct flow. Still, the exact details of the inflow profiles can be ignored. The Mach number is of the order of 0.5 , so that a compressible solution is imperative. The temperature is virtually constant (dropping by $2 \%$ ) but the Mach number rises significantly along the duct, as needed to maintain the mass flow while the pressure is dropping. In that sense, there is no "fully-developed state." The walls are treated as adiabatic, since the GFIT air supply is heated to the adiabatic wall temperature during a run.

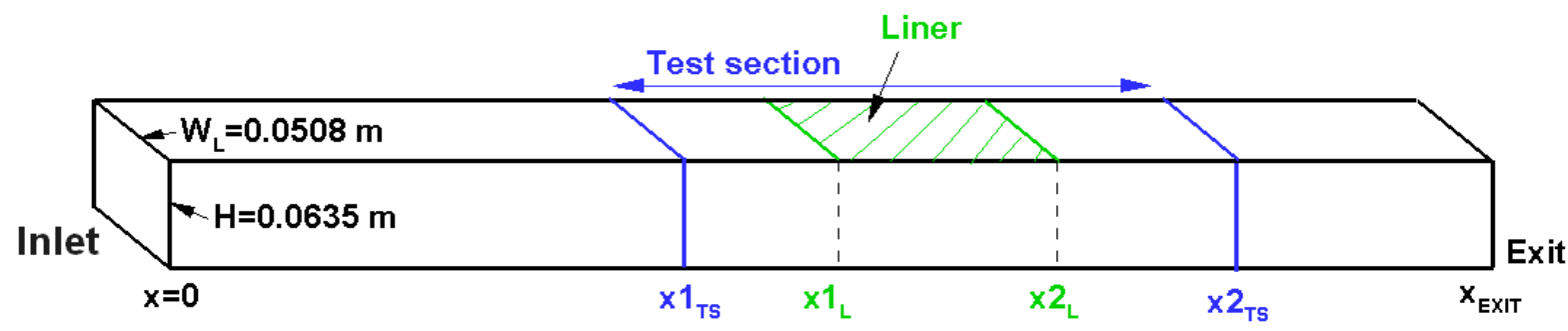

Figure 1. Computational domain, reproducing the test installation.

As mentioned previously, the duct is nearly square, and three of the walls remain smooth. A portion of the upper wall, between $x 1_{L}=4.05 \mathrm{~m}$ and $x 2_{L}=4.49 \mathrm{~m}$, is used to simulate the additional effective roughness of an acoustic liner. The roughened length is not large compared with the duct depth, so that transient effects are expected as the higher skin friction of the acoustic liner propagates toward the core region of the velocity field. In addition, the reduced velocity along this portion and conservation of mass raise the velocity near the smooth walls, and therefore the skin friction. This could introduce a spurious increase in pressure drop, which is not actually associated with the liner and depends on the duct size. All these effects are rendered by a CFD solution, and being simple effects of the momentum equation, are not presumed to be particularly vulnerable to the imperfections in turbulence modeling.

The measurements center on the Duct Resistance Factor $\lambda$, defined by 


$$
\lambda=-\frac{d p}{d x} \frac{d_{h}}{q}
$$

where $d p / d x$ is the pressure derivative in the streamwise direction measured by the static pressure drop over the test section $\left[x 1_{T S}, x 2_{T S}\right]=[3.80 \mathrm{~m}, 4.87 \mathrm{~m}]$ which brackets the rough section, $d_{h}$ the hydraulic diameter of the duct, and $q$ the reference dynamic pressure.

The initial formula (5) in [1] used there to relate $\lambda$, as measured, and the skin friction $\lambda_{L}$ of the liner compared with the skin friction $\lambda_{S W}$ of the smooth wall can be presented in the form:

$$
\left[\frac{\left(C_{f}\right)_{L}}{\left(C_{f}\right)_{S W}}\right]_{\exp t} \equiv\left(\frac{\lambda_{L}}{\lambda_{S W}}\right)=1+\frac{P}{W_{L}} \frac{S}{L L}\left(\frac{\lambda_{L+S W}}{\lambda_{S W}}-1\right)
$$

Here, $\lambda_{L+S W}$ in the right hand side is measured with one lined area and three smooth walls, and $\lambda_{S W}$ is the baseline measurement using a smooth wall sample. $W_{L}$ is the liner width and $P$ the duct perimeter. $L L$ is the liner length, and $S$ the distance between the pressure ports used to define $d p / d x$.

This formula would be exactly valid under a set of many conditions: a homogeneous flow in $x$, without interference between the liner and the smooth walls, and other requirements which are not exactly satisfied in the present rig, for instance the existence of a potential core with a single dynamic pressure, $q$. Determining how accurate an approximation (2) is in the GFIT runs is the first motivation of the present study.

Conventional turbulence modeling is used, and well validated by comparisons with experiment. The Quadratic Constitutive Relation (QCR) terms in RANS models are desirable, as this type of duct generates corner vortices, although not crucial. The RANS turbulence model is the SA-QCR model, [5-6] with the roughness term discussed by Aupoix \& Spalart [4], and by the NASA Turbulence Modeling Resource [7]. The NTS Navier-Stokes code is used [8], with $3^{\text {rd }}$-order upwind-biased Roe scheme for the approximation of the inviscid fluxes and $2^{\text {nd }}$-order centered scheme for the viscous terms. To match the experimental conditions, the stagnation temperature at the inlet is prescribed, whereas the inlet Mach number and the downstream static pressure are adjusted to obtain the desired Mach number $\mathrm{M}=0.5$ at the centerline of the exit section $x=7.1 \mathrm{~m}$ and a static pressure equal to the atmospheric pressure $p_{s}=p_{a t m}$ in the middle of the test section (at $x=4.33 \mathrm{~m}$ ).
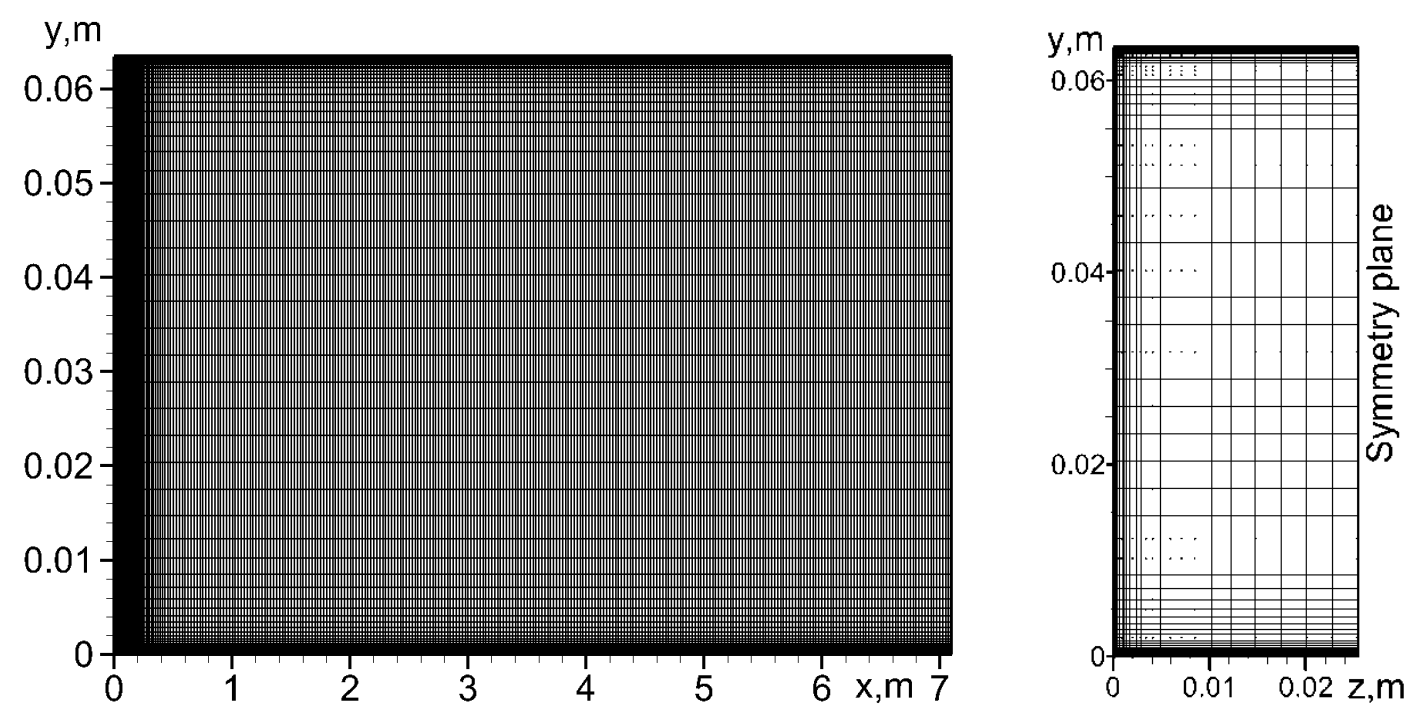

Figure 2. Numerical grid. Note different scales for $x$ and $y$ in the left frame. 
The grid has 332 by 93 by 45 points, as shown in Fig. 2 . The symmetry in the z direction allowed a grid count of 45. This grid obeys the standard guidelines for turbulent wall-bounded flows, and ensured virtually grid-independent solutions.

\section{Baseline Results}

We begin with an overall description of the flow, and an experimental validation for the duct with smooth walls. Figures 3 and 4 illustrate the strong inhomogeneity of the flow in the streamwise direction, mentioned earlier. The entry region, up to $x \sim 2.2 \mathrm{~m}$, is dominated by the growth of the boundary layers, which accelerates the core flow roughly from $\mathrm{Mach}=0.35$ to $\mathrm{Mach}=0.45$. The Mach number then has a plateau, before rising again as the density and speed of sound gradually drop in the fully-turbulent regime. The pressure drop over the test section is $3310 \mathrm{~Pa}$, of which the skin friction accounts for $2636 \mathrm{~Pa}$ and the increase in momentum flux for $682 \mathrm{~Pa}$. In other words, an assessment of the skin friction by the pressure drop results in an over-estimation by a full $25 \%$, as illustrated in Fig. 3. These phenomena are not reflected at all by the pressure distribution, which descends almost linearly from entry to exit.

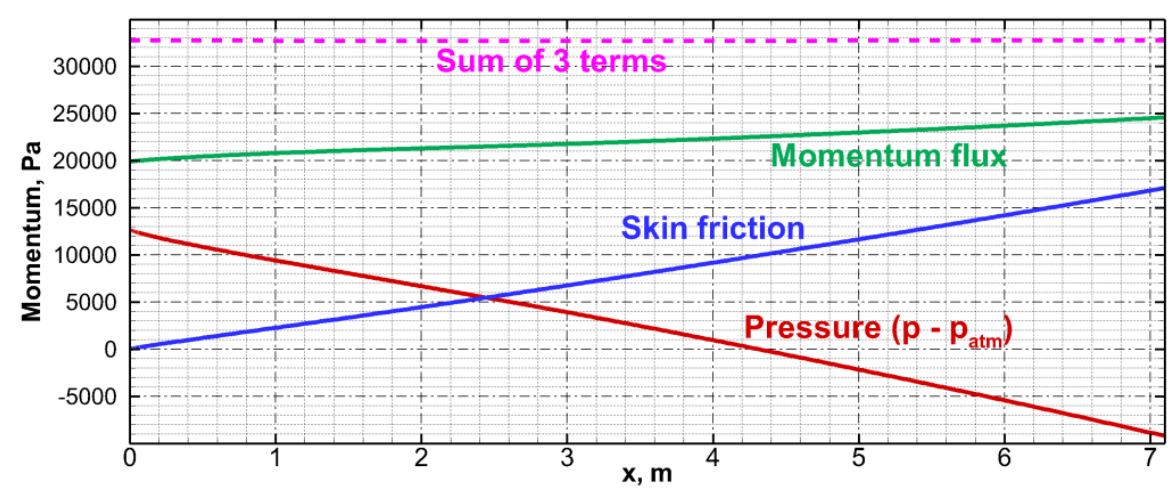

Figure 3. Distributions of the terms in the integral momentum balance along the duct.

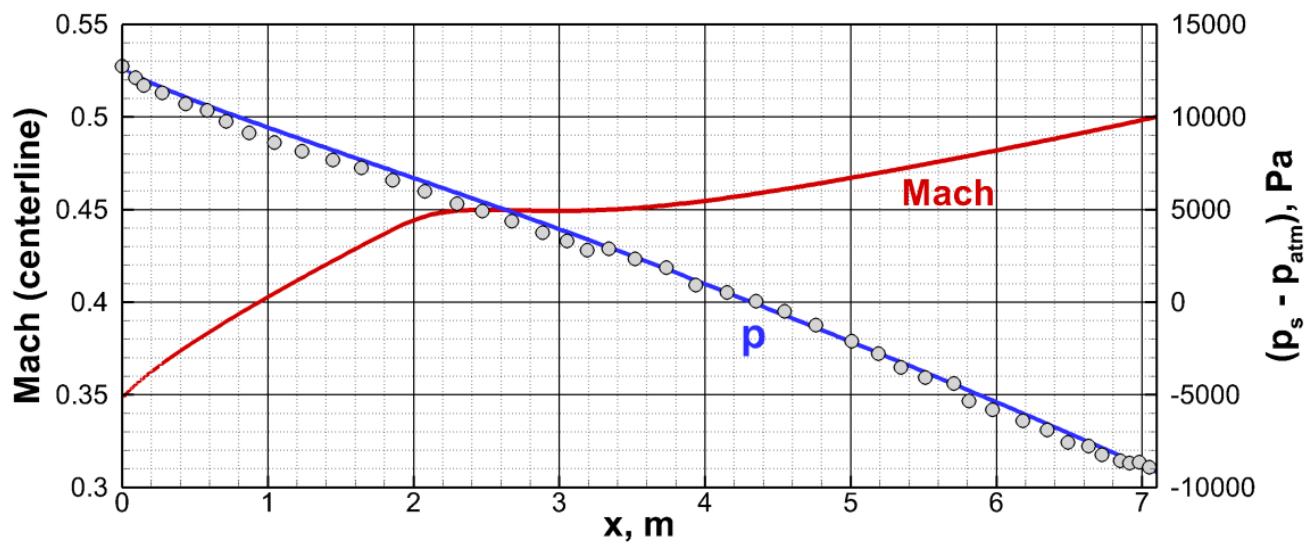

Figure 4. Distributions of pressure and Mach number along the duct. Lines: CFD; Symbols: experiment [1].

Figure 4 displays excellent agreement between test and CFD over the pressure distribution, which is most encouraging regarding the performance of the turbulence model, the code, the grid, and the boundary conditions used. The numerical system appears ready for cases with skin-friction variations. 


\section{Results with Simulated Roughness}

After the successful baseline comparison, a case was run with a rough wall, not yet adjusted to reproduce the acoustic liner effect quantitatively, but simply to produce a measurable skin-friction increase. The equivalent sandgrain size is $k_{s}=1.510^{-4} \mathrm{~m}$, using Nikuradse's sand-grain size convention [9], to be compared with the duct width of about $0.05 \mathrm{~m}$
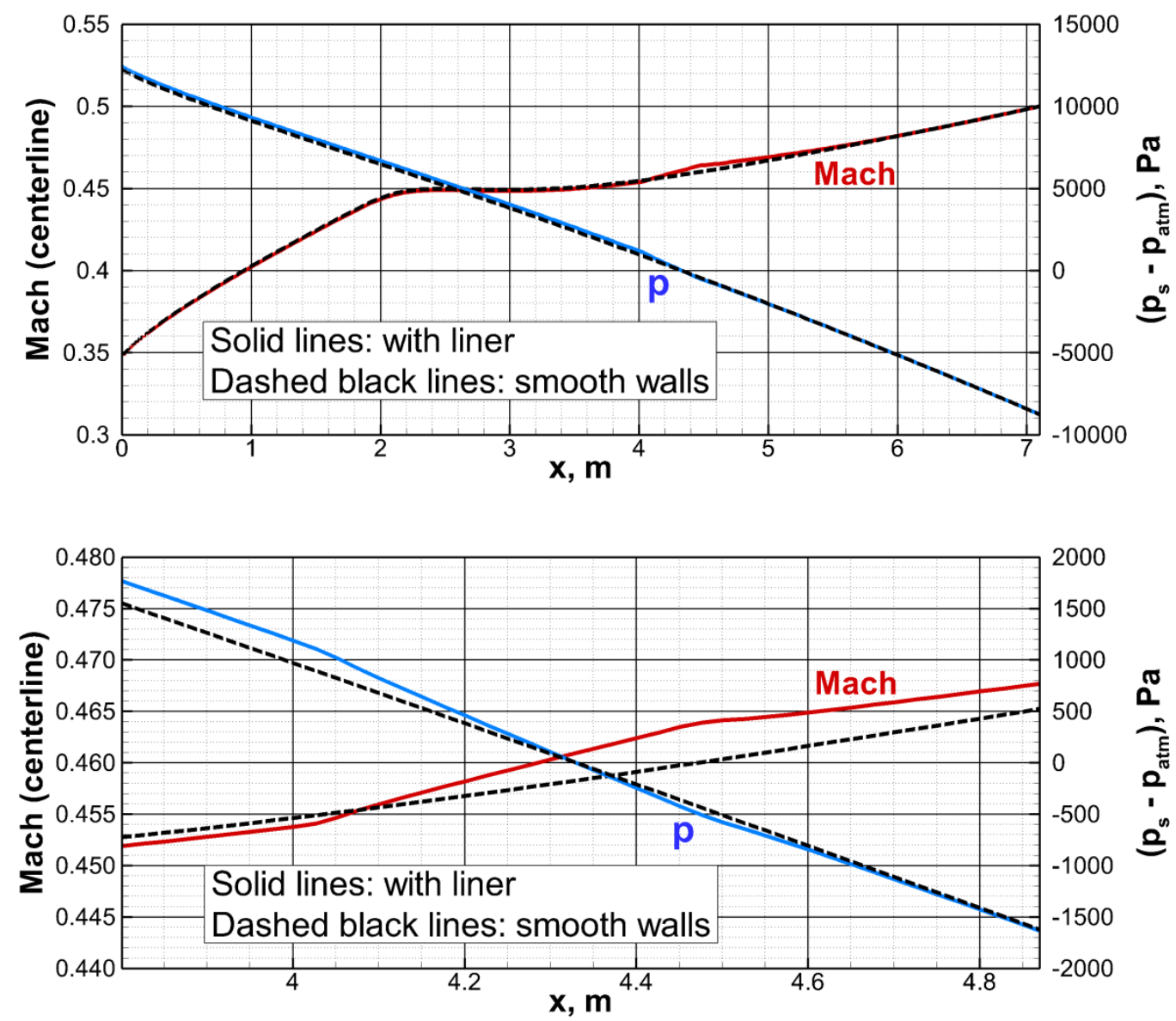

Figure 5. Effect of liner on distributions of pressure and Mach number along the duct. Top: global view; bottom: close-up view within test-section.

Figure 5 presents a comparison of distributions of the Mach number and static pressure along the duct, with and without liner in place. It reveals an increase of the pressure drop associated with the liner and shows that even at the rather large (see below) value of the grain size considered in this initial test, this increase is small compared to the baseline pressure drop itself. 


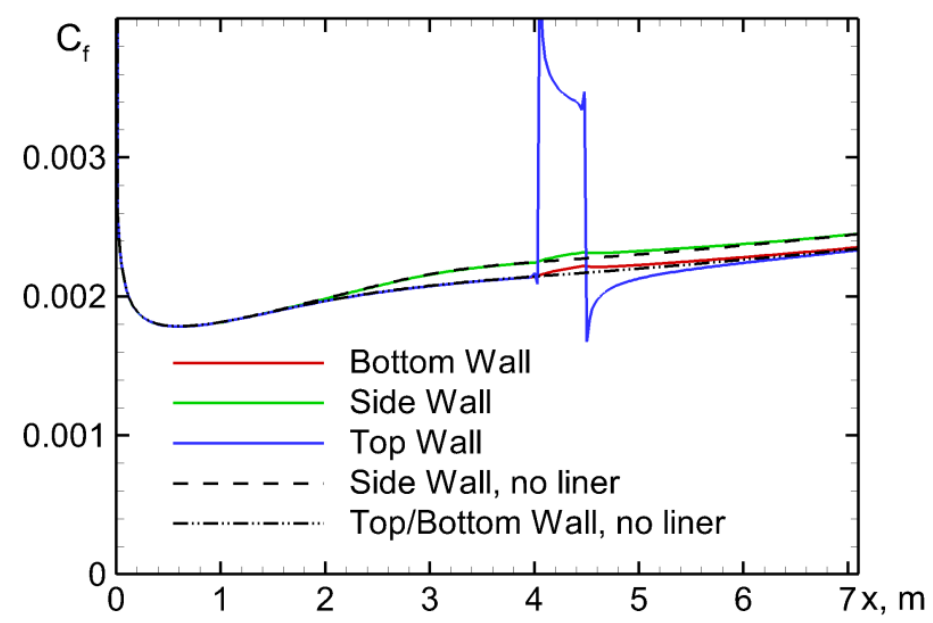

Figure 6. Skin friction on all walls, with and without liner in place. Global view.

In contrast, in Figure 6, displaying the entire skin-friction distribution along the centerline of each wall, the sudden increase in skin friction is very visible, as is the transient effect. In fact the transient effect is present over the entire rough wall section, in that the spike is followed by a relaxation that still gives a negative slope at the end of the roughened area, a slope which is opposite to the general upwards trend. A similar transient follows the return to a smooth wall, and at the exit, the residual effect of the rough patch has decayed considerably. Thus the finite length of the rough area causes transients both at its leading and trailing edges, which largely cancel each other in the static pressure drop measurement. The slight asymmetry of the baseline flow, with higher skin friction on the side walls than the top and bottom walls and due to the rectangular cross-section, is also noted.

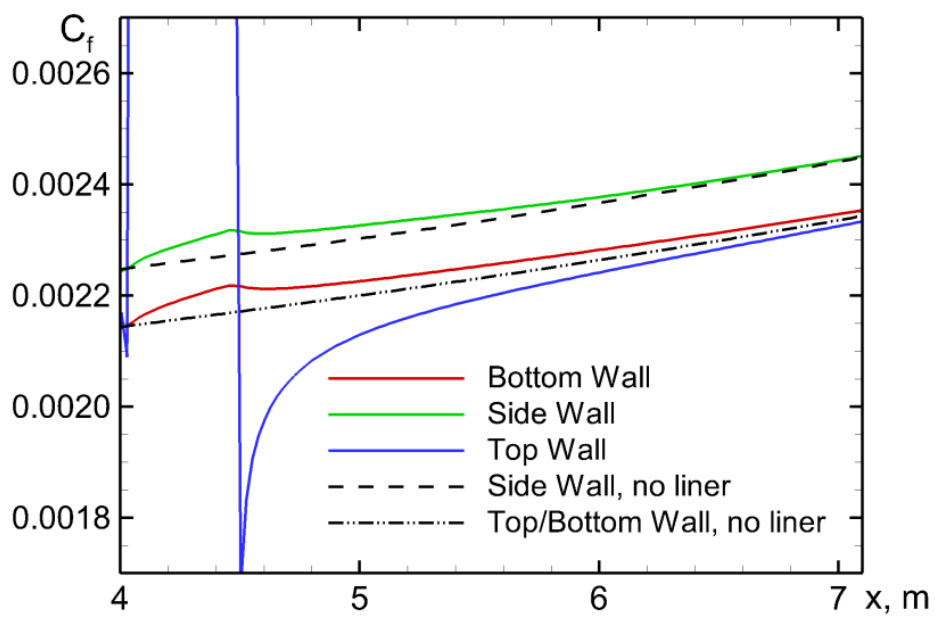

Figure 7. Skin friction on all walls. Close-up view.

Figure 7 focuses on the region from the start of the roughened area to the end of the computational domain. The reduced scale reveals the blockage effect, namely how the skin friction on the smooth walls increases in response to the simulated roughness, although only by a few percent. The rough-wall skin friction is discontinuous, but the smooth-wall skin friction only has a jump in slope. This effect is also marked by a transient, and is still developing at the end of the roughened area. It is also very persistent, with effects beyond the end of this area. 

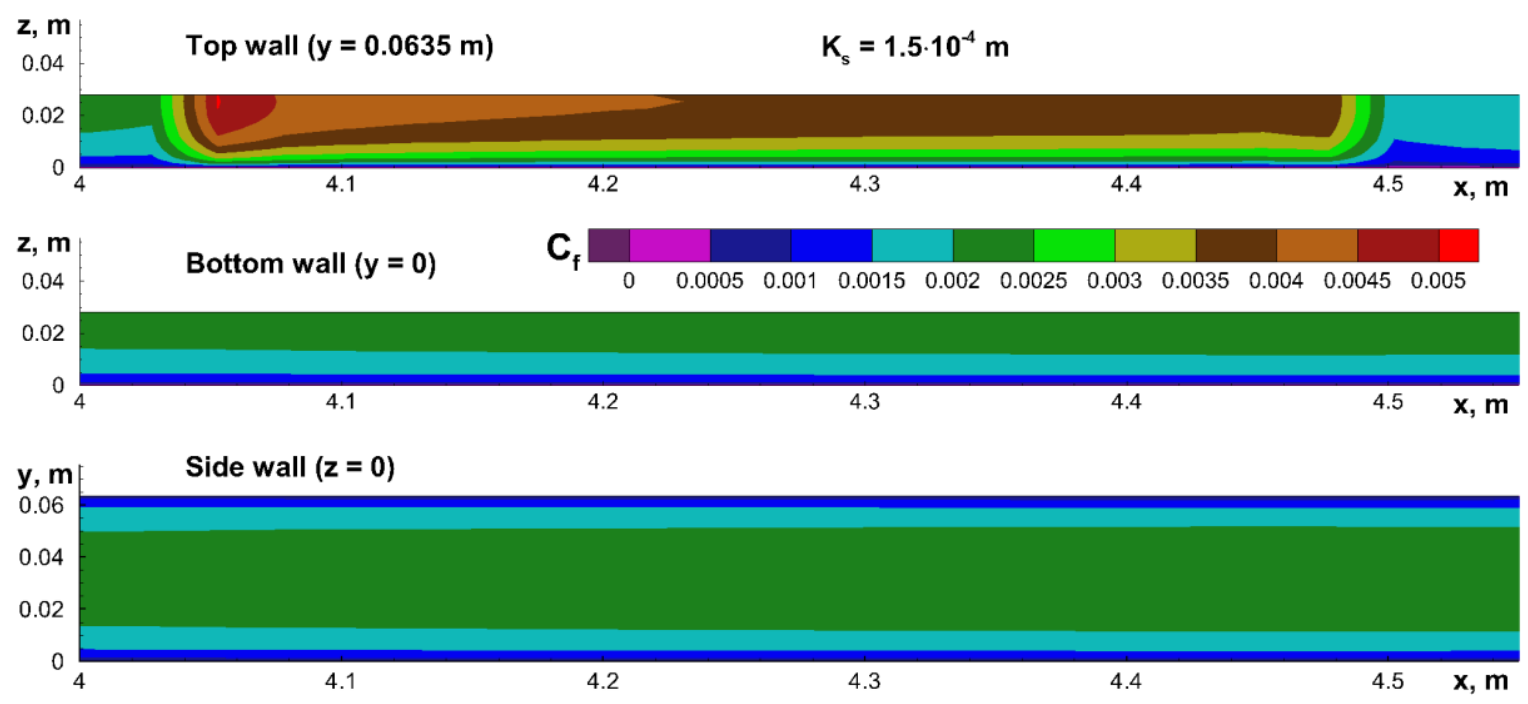

Figure 8. Skin-friction distributions on all walls.

Finally, Fig. 8 illustrates features of the flow away from the centerline. The calculations only need to resolve half of the flow domain, by symmetry. The skin friction drops to zero in the corners, naturally, although this tendency is mitigated by the corner vortices, which the QCR model reproduces fairly well [5,6]. The lateral dependence appears much stronger over the liner than over the smooth walls, creating a non-trivial interference that is hidden by the centerline profiles in Figs. 6 and 7. The transient effect is also very visible. All these effects are ignored by the basic $\lambda$ equation, above. We now explore the quantitative consequences of these effects.

For this purpose the computation was repeated with the rough patch extended to the end of the computational domain, and the friction coefficients on the rough and smooth walls were compared, after the transient is complete. As illustrated by Fig. 9, the corresponding skin friction coefficient on the rough wall turns out to be 1.42 times higher than that in the duct without the rough patch. This appears to be the most meaningful measure of the effect of the wall conditions. However (1), applied between the two pressure ports, and a subsequent calculation of the friction increase with the use of (2) give for the considered case $\lambda_{L+S W} / \lambda_{S W} \approx 1.072$ and $\lambda_{L} / \lambda_{S W} \approx 1.79$. Thus our CFD study suggests that the simple equation (2) overestimates the absolute skin friction ratio $\left(C_{f}\right)_{L} /\left(C_{f}\right)_{S W}$ by around $25 \%$ and the skin-friction penalty $\left[\left(C_{f}\right)_{L} /\left(C_{f}\right)_{S W}-1\right]$ by almost $90 \%$. The "reverse" transient seen in Fig. 6 after the removal of the roughness is insufficient to compensate. This is a very large effect, which should be taken into account, although it would not invalidate the ranking of different liners, so that design decisions would be correct.

Note that this transient effect and the danger of over-estimating the changes due to using relatively short samples of drag-reducing or drag-increasing conditions was already pointed out for riblets [10]. 

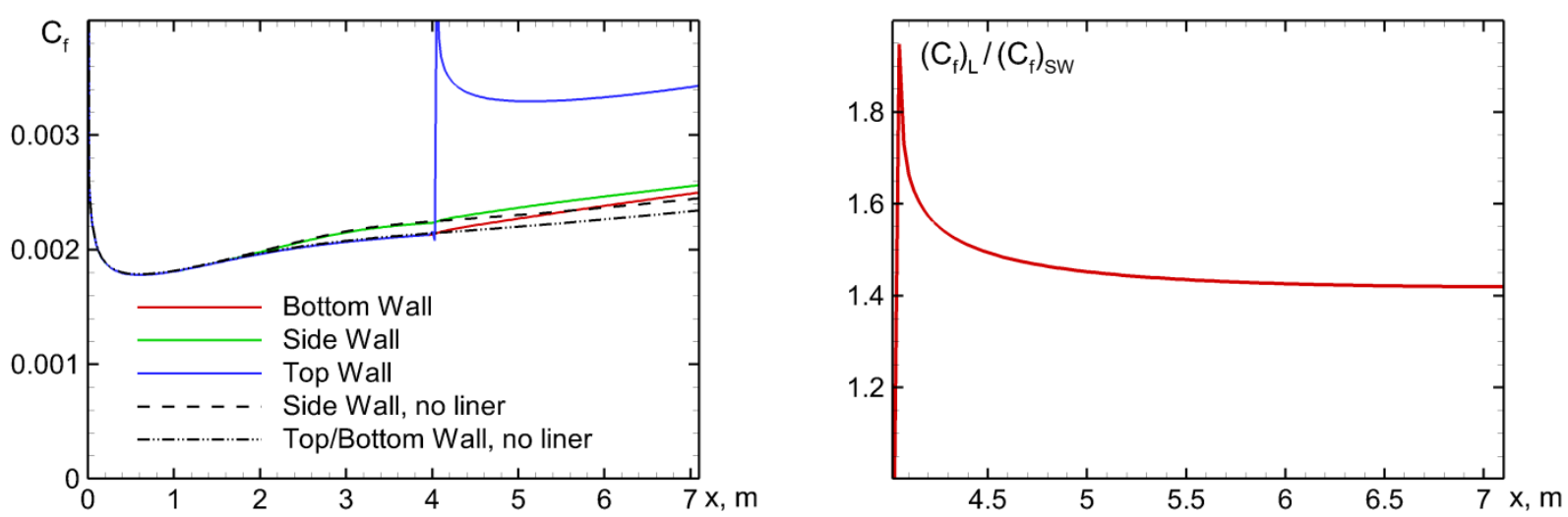

Figure 9. Skin friction with long patch, beyond transient region (left) and ratio of skin friction coefficients on rough and smooth walls (right).

\section{Calculation of the Effective Roughness Length}

Here we consider the "conventional liner" from [1], for which the declared increase in resistance factor $\lambda_{\mathrm{L}} / \lambda_{\mathrm{SW}}$ at Mach $=0.5$ is $8 \%$ (Fig. 9 of [1]). Then, applying (5) of that paper ((2) of the present paper) in reverse with the dimensions of the apparatus, we obtain for the ratio of the measured pressure drops $\lambda_{\mathrm{L}+\mathrm{SW}} / \lambda_{\mathrm{sw}}$ the value 1.0076 (the difference between this number and 1 is smaller than 0.08 by about an order of magnitude, due to only one wall being altered, and the patch being much shorter than the distance between pressure ports). This is a much smaller ratio than what our nominal roughness value gave, namely 1.08 (in hindsight, this may not be surprising, in that our guess resulted in a drastic increase in skin friction, which typical designs probably avoid). Therefore, the calculation was repeated, and the Nikuradse sand-grain size adjusted [1]. The final value is $\mathrm{k}_{\mathrm{s}}=210^{-5} \mathrm{~m}$. This is the first estimate of the roughness value that would be applied for this surface in a viscous CFD solution for an airplane. The sand-grain roughness in wall units is $\mathrm{k}_{\mathrm{s}}{ }^{+} \sim 8$, and therefore transitionally rough. At cruising altitudes, the velocity will be higher than here, but the molecular viscosity is about double, so that the roughness expressed in wall units will be similar.

The area of the fan-duct acoustic liner, based on a cursory examination of a Boeing 787 drawing, is about $1 / 3$ of the wing area. Therefore, an $8 \%$ increase in skin friction would have a non-negligible impact on fuel burn, of a few tenths of $1 \%$. A thorough representation of the skin friction in the fan duct has actually not been achieved to our knowledge, because with the fan and guide vanes the turbulence level is quite high in addition to the acoustic level, and current CFD methods do not include either effect on the boundary-layer turbulence and therefore the skin friction.

The calculation was repeated for a case with $140 \mathrm{~dB}$ and then $150 \mathrm{~dB}$ acoustic excitation, following [1]. At the considered Mach number $\mathrm{M}=0.5$, the $140 \mathrm{~dB}$ excitation did not alter the equivalent roughness, but with $150 \mathrm{~dB}$, using Fig. 10 the equivalent roughness rises to $\mathrm{k}_{\mathrm{s}}=3.510^{-5} \mathrm{~m}$. 


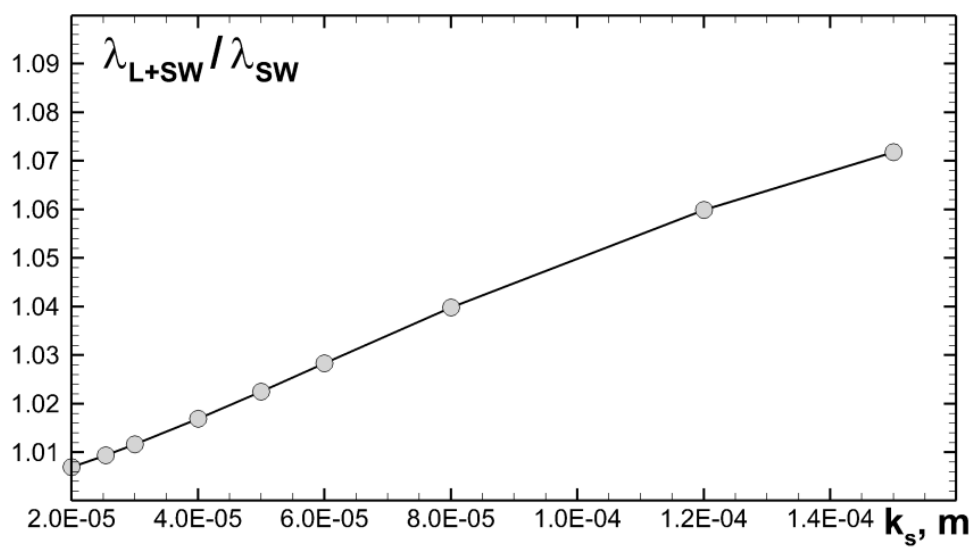

Figure 10. Variation of the ratio of pressure drops over test section with and without rough patch with variation of sand-grain size.

Finally, Figure 11 displays the apparent penalty caused by a relatively short rough patch, and that caused by a long patch, which would be more realistic, certainly for an airplane's fan duct. The difference is quite significant, in the direction of over-estimating the penalty.

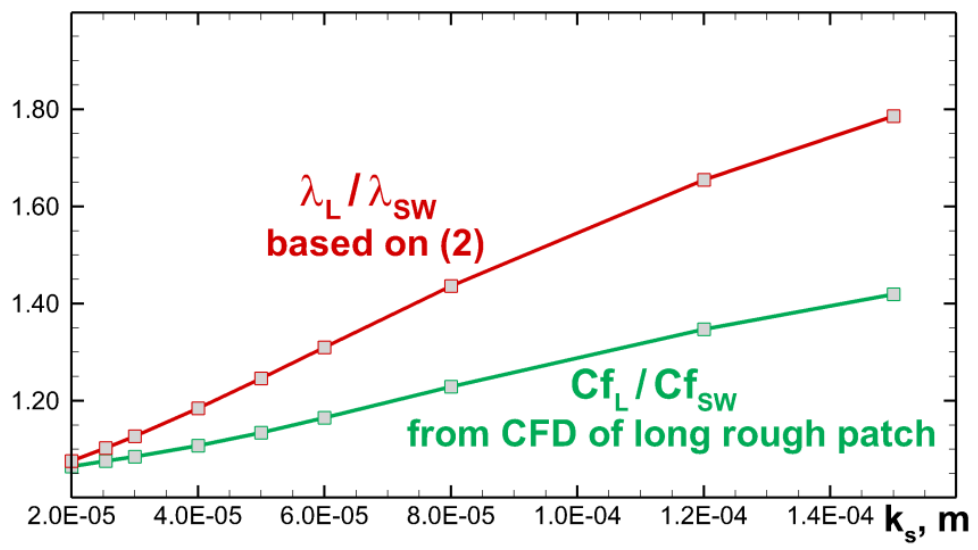

Figure 11. Effect of sand-grain size on increase of skin-friction coefficient relative smooth wall obtained in CFD of duct flow with long rough patch and computed by (2) based on the pressure drop over the test section.

\section{Concluding Remarks}

Our CFD study, based on conventional RANS turbulence modeling, of the NASA Langley Grazing Flow Impedance Tube appears conclusive, and to provide quantitative information. The principal conclusion is that the simple equation (2) which has been used to deduce the skin friction from the pressure drop overestimates the skinfriction absolute value by about $25 \%$ and the relative penalty $\left(\lambda_{L} / \lambda_{S W}-1\right)$ by nearly $90 \%$, which is significant enough to be taken into account. This is due to both the sustained inhomogeneity of the flow as the pressure drops and the momentum flux steadily increases, which the formula ignored, and to transient effects as the effective roughness is imposed, and then removed. On the other hand, this effect would not invalidate the ranking of different surfaces, so that design decisions would be correct. The study also provides the Nikuradse equivalent sand-grain roughness, which is found to be about $\mathrm{k}_{\mathrm{s}}=210^{-5} \mathrm{~m}$ for the conventional perforated acoustic liner without sound, and $\mathrm{k}_{\mathrm{s}}=3.510^{-5} \mathrm{~m}$ with an acoustic excitation at the $150 \mathrm{~dB}$ level. These would be the tentative values introduced into a Navier-Stokes solution for the aircraft, with the purpose of assessing the true impact of the liners on performance. 


\section{Acknowledgments}

Drs. M. Shur and M. Strelets assisted in the computations and analysis. The authors would like to thank Carol Harrison of NASA Langley for her efforts in obtaining the experimental data.

\section{References}

[1] Howerton, B. M., and Jones, M. G. "Acoustic Liner Drag: Measurements on Novel Facesheet Perforate Geometries." AIAA 2016-2979.

[2] Howerton, B M., and Jones, M G. “Acoustic Liner Drag: A Parametric Study of Conventional Configurations,” AIAA 2015-2230.

[3] Spalart, P. R. and Allmaras, S. R. "A one-equation turbulence model for aerodynamic flows." La Recherche Aerospatiale, 1, 1994, pp. 5-21.

[4] Aupoix, B. and Spalart, P. R. 2003. "Extensions of the Spalart-Allmaras turbulence model to account for wall roughness." Int. J. Heat Fluid Flow 24, pp. 454-462.

[5] Spalart, P. R., "Strategies for Turbulence Modeling and Simulation,” Int. J. Heat Fluid Flow 21 (2000) pp. 252-263.

[6] Mani, M., Babcock, D. A., Winkler, C. M., and Spalart, P. R. "Predictions of a Supersonic Turbulent Flow in a Square Duct," AIAA 2013-0860.

[7] Rumsey, C. L., “Turbulence Modeling Resource,” https://turbmodels.larc.nasa.gov [retrieved 2017].

[8] Shur, M., Strelets, M., Travin, A. "High-order implicit multi-block Navier-Stokes code: Ten-year experience of application to RANS/DES/LES /DNS of turbulent flows," $7^{\text {th }}$ Symposium on Overset Composite Grids \& Solution Technology, Huntington Beach, CA, 2004. http://cfd.spbstu.ru/agarbaruk/c/documentlibrary/DLFE-42505.pdf [retrieved 2017].

[9] Nikuradse, J., “Laws of Flow in Rough Pipes,” NACA TM-1292, November 1950.

[10] Spalart, P. R., McLean, J. D., "Drag reduction: enticing turbulence, and then an industry,” Phil. Trans. R. Soc. A (2011), 369, 1556-1569. 